\title{
Políticas sociales y jóvenes. Aportes desde los derechos humanos
}

\section{Social policies and youth. Contributions from human rights}

\author{
Yussef Becher yussefbe@hotmail.com \\ http://orcid.org/0000-0003-1256-3890 \\ Universidad Nacional de San Luis/ \\ Consejo Nacional de Investigaciones \\ Científicas y Técnicas (Argentina)
}

\section{Resumen}

Al cumplirse quince años de los Objetivos de Desarrollo del Mileno (ODM), los miembros de la Organización de Naciones Unidas (ONU) establecieron una nueva agenda -mediante los Objetivos de Desarrollo Sostenible (ODS)- en donde reiteraron la importancia de incorporar el marco de los derechos humanos en las estrategias de desarrollo a fin de reducir desigualdades sociales. Entre tales estrategias quedan comprendidas las políticas sociales y las que tienen como destinataria a la juventud en tanto sector socio demográfico.

La incorporación del enfoque de derechos humanos supone una modificación en el modo tradicional de concebir las intervenciones sociales y, al mismo tiempo, la incorporación de un conjunto de estándares -elaborados por los grupos de trabajo dedicados al tema- que garantizan mayor universalidad y, por consiguiente, reducción de desigualdades sociales. 
A fin de reflejar la experiencia argentina en tales ámbitos, seleccionamos dos casos de estudio integrados por programas sociales con presencia juvenil significativos por diferentes motivos. En un caso se trata de una acción estatal nacional -el Programa de Respaldo a Estudiantes Argentinos- que ha obtenido una importante masividad a nivel nacional y, en el otro, de un programa social de la provincia de San Luis respecto de la cual, dado que se trata del interior del país, son pocos los estudios que obtienen difusión en comparación con la Ciudad de Buenos Aires y el área Metropolitana.

Palabras clave: Políticas sociales; juventudes; enfoque de derechos humanos; Programa de Respaldo a Estudiantes Argentinos.

\section{Abstract}

On the fifteenth anniversary of the Millennium Development Goals (MDGs), the members of the United Nations (UN) established a new agenda -through the Sustainable Development Goals (SDGs)- where they reiterated the importance of incorporating the framework for human rights in development strategies to reduce social inequalities. Such strategies include social policies and those aimed at youth as a socio-demographic sector.

The incorporation of the human rights approach implies a modification in the traditional way of conceiving social interventions and, at the same time, the incorporation of a set of standards elaborated by the working groups dedicated to the subject- that guarantee greater universality and, consequently, reduction of social inequalities.

In order to reflect the Argentine experience in these areas, we selected two case studies integrated by social programs with significant youth presence for different reasons. In one case, it is a national state action -the Programa de Respaldo a Estudiantes Argentinos- that has obtained an important massively national level and, in the other, a social program of the Provincia de San Luis regarding which, given that it deals with the interior of the country, there are few studies that obtain diffusion in comparison with the Ciudad de Buenos Aires and the Área Metropolitana.

Keywords: Social policies; youth; human rights approach; Programa de Respaldo a Estudiantes Argentinos. 
En el marco de los Objetivos de Desarrollo del Milenio (ODM) se propuso particularmente la incorporación de los derechos humanos en las estrategias de desarrollo. Entre dichas estrategias a las políticas sociales les corresponde una importante tarea, pues son las encargadas de la redistribución del ingreso, a partir de lo cual pueden contribuir a la reducción de desigualdades sociales. Ese mismo es el propósito número 10 planteado quince años después en los Objetivos de Desarrollo Sostenible (ODS). En tal sentido, en el texto mostraremos la incidencia que sobre tales desigualdades puede tener la incorporación del enfoque de derechos humanos en las políticas sociales. Para ello consideramos que el principio de universalidad en materia de intervenciones sociales contribuirá a un desarrollo social inclusivo, en donde se eliminarán situaciones estructurales de discriminación entre las que se comprenden las dirigidas a las mujeres. Ahora bien: ¿Por qué dedicarnos a políticas sociales de juventud? Sobre dicho colectivo han recaído históricamente, dadas particulares condiciones materiales y simbólicas, mayores situaciones de vulnerabilidad. En estos aspectos los derechos humanos vinculados con las juventudes -en el marco de las políticas socialespueden constituir un aporte relevante para superar esas dificultades.

A fin de reflejar la experiencia argentina, desde ya parcialmente, vamos a tomar en cuenta dos casos de estudio de programas sociales juveniles: el Programa de Respaldo a Estudiantes Argentinos (PROGRESAR) y el Plan de Inclusión Educativa (PIE). Tales casos resultan significativos por varios motivos. El primero es un programa nacional y el otro provincial. Ello agrega un interesante elemento de análisis por cuanto -tal como señalan los estudios en el área- por lo general las investigaciones sociales se han centrado principalmente en la Ciudad de Buenos Aires y el Área Metropolitana. EI PIE es un programa implementado en la provincia de San Luis, donde se desarrollan las actividades de investigación, que presenta notables semejanzas con el PROGRESAR pues ambos tienen como objetivo la terminalidad educativa por medio del acceso al derecho a la educación de quienes son sus jóvenes destinatarios. Tales semejanzas son las que hacen posible la comparación y el diferente escenario social en donde se implementan la enriquece, pues no sólo se agregan aspectos geográficos sino otros propiamente simbólicos.

Dado que vamos a indagar dos de las etapas que conforman a las políticas sociales -diseño e implementación- procuramos adoptar una serie de particularidades teórico-metodológicas en donde prima un enfoque transdisciplinar. En el estudio de los diseños tendremos en cuenta los documentos oficiales y en su implementación, las percepciones y experiencias de sus destinatarios respecto del acceso a la información sobre los programas, lo cual repercute sobre la universalidad y masividad, y las instancias de reclamo. En ambos aspectos se adopta un 
enfoque cualitativo y se acude al trabajo empírico iniciado en 2016 y en desarrollo en el marco de una tesis doctoral inserta en un proyecto de investigación de la Universidad Nacional de San Luis (UNSL). Los resultados muestran que tales programas no incorporan en su diseño en su totalidad el enfoque de derechos humanos, pues acuden a criterios de focalización y presentan ausencia de instancias de participación juvenil. Al mismo tiempo, en su implementación en los aspectos que comprende al enfoque de derechos, acceso al programa y mecanismos de reclamo, incide particularmente el plano de lo vincular -mediante redes de sociabilidad- y aspectos territoriales propios de la provincia.

\section{Desigualdades y políticas sociales}

Los estudios contemporáneos sobre el tema han señalado la necesidad de pluralizar la noción a la que referimos en el título por cuanto son múltiples los factores que inciden en los procesos de construcción de desigualdades sociales. Entre dichos factores pueden incorporarse aspectos territoriales, como así también otros referidos al medio ambiente y el reconocimiento de la diversidad. Por solo mencionar algunos polos de desigualdad que se han incorporado recientemente.

Durante muchos años fue la variable económica la que predominó en la conformación del concepto, pero dada la mundialización -con la incidencia del mercado de consumo y la sociedad de la información- y la heterogeneidad que son propias de la vida cotidiana, fue necesario incorporar otros elementos en su estudio. Al respecto afirma Levitas (2006) la dificultad para abordar tales aspectos desde perspectivas cuantitativas y lo imperioso de incorporar miradas cualitativas.

El concepto de desigualdad en tanto tal comienza a emerger a partir de estudios sociales en Europa en la década de 1970. Entre los aportes señeros se destacan el de Lenoir (1974) y el de Towsend (1979). En el primero de ellos se describe la situación de los ciudadanos franceses excluidos de la protección social y, en el segundo, se propone un concepto de pobreza en donde no solo se tengan en cuenta consideraciones objetivas sino también condiciones de vida a nivel subjetivo. Entre las contribuciones teóricas y empíricas más recientes con difusión a nivel mundial, que abordan la categoría desde la complejidad que le es propia, podemos mencionar aquellas que provienen de la sociología francesa e inglesa dedicada al tema. En la primera línea se ubica como uno de sus principales referentes a Castel y en la segunda a Levitas a quien ya hemos citado. Si bien existen algunas divergencias entre unos y otros 
planteos teóricos, es factible encontrar muchos puntos en común. Tanto unos como otros coinciden en que las exclusiones son graduales y las definen en términos procesuales: nadie puede estar excluido de un momento a otro. Dice Autés:

\begin{abstract}
Estos tres enfoques consideran que la exclusión, como quiera que la llamen, es un proceso... en S. Paugam es una estrategia de etiquetado de los individuos por parte de las instituciones, de categorización; en V. de Gaulejac, de análisis centrados en los destinos individuales, incluidos los fenómenos de subjetivación y desubjetivación; en R. Castel, de las etapas de un proceso histórico y del tratamiento de la cuestión social por el Estado (2004: 32).
\end{abstract}

Por su parte Estivill (2003) señala: "La exclusión social puede ser entendida como una acumulación de procesos confluyentes con rupturas sucesivas que surgen desde el corazón de la economía, la política y la sociedad" (p. 19, traducción propia).

El mismo Castel (2004) cuando define las zonas de la vida social lo hace a partir de dos elementos -el empleo y los soportes de sociabilidad- colocando a la exclusión como el último estadio al cual llega el sujeto cuando pierde vínculos con el empleo y se debilitan sus redes de sociabilidad. El último es un aspecto que preocupa a las dos tradiciones teóricas por cuanto consideran que los procesos de desigualdad debilitan el lazo social. De Gaulejac y TaboadaLeónetti (1994) sostienen que los procesos exclusivos afectan la estructura identitaria de los sujetos por cuanto auto perciben que ya no existe para ellos un lugar en la sociedad. Los ingleses agregan a esta cuestión los efectos sobre la construcción de ciudadanía pues -tal como señalan Burchardt, Le Grand y Piachaud (2002)- las desigualdades obturan el involucramiento en actividades sociales que exceden las posibilidades de los propios sujetos, aunque tengan intención de participar. Quienes experimentan condiciones de pobreza a nivel objetivo en la Argentina suelen tener serias dificultades para el ejercicio de derechos tales como la libertad de asociación y la iniciativa popular como modalidades de reclamo ante la administración pública (Abramovich, 2006). Al mismo tiempo, las mujeres argentinas se encuentran frente a dificultades para el ejercicio de derechos civiles y políticos dado el desconocimiento estatal de las tareas de cuidado que asumen y les han sido asignadas respondiendo a estereotipos sociales (Pautassi, 2007).

Ahora bien: ¿Qué papel les corresponde a las políticas sociales? Tales intervenciones estatales son definidas como aquellas que se orientan, en el sentido que producen y moldean, a las condiciones de vida y de reproducción de la vida de un determinado sector de la sociedad, y lo hacen especialmente en el momento de la distribución secundaria del ingreso 
(Danani, 2004). Como bien señala la definición, sus efectos recaen principalmente sobre las condiciones de vida de la población -en la contemporaneidad, un sector socio demográfico pues tienden a ser focalizadas- y por consiguiente pueden tener una importante incidencia sobre la igualdad de tales condiciones entre unos y otros sectores. Por ello, tal como afirma el sociólogo francés Dubet (2011), no es indistinta en el marco de las políticas sociales la opción por la igualdad de oportunidades o la de posiciones. La primera de ellas -la cual discursivamente ha tenido mayor performatividad- propone la igualdad de oportunidades para todos los ciudadanos sin considerar las diferentes condiciones de unos y otros. De modo que en dicha igualdad lo que finalmente prima es la meritocracia. Por su parte, la igualdad de posiciones propone la reducción o eliminación de aquellos condicionantes que obturan la paridad de posibilidades entre unos y otros sectores sociales. Dicha igualdad resulta significativa en la disminución de desigualdades de género, especialmente, en lo que la bibliografía sobre el tema ha denominado techos de cristal.

\section{La propuesta del enfoque de derechos humanos}

Ya hemos mencionado que en el marco de los ODM se propuso particularmente la incorporación del marco que ofrecen los derechos humanos -plasmados en diferentes instrumentos internacionales- en las estrategias de desarrollo. Entre tales estrategias quedan comprendidas las políticas sociales con las finalidades que antes hemos descripto. Quince años después en los ODS esta misma propuesta continúa presente.

\section{Los estándares para políticas sociales}

A fin de incorporar el enfoque de derechos humanos en las acciones estatales con fines redistributivos se proponen una serie de estándares tanto formales como sustanciales (1). Ello supone que no solo deben estar presentes, como suele suceder, en la instancia de diseño de una política sino también en la de su implementación. Por ello, se señala que el enfoque debe guiar todo el proceso de formulación de una intervención social por cuanto se considera a ese término, el de formulación, como comprensivo de las dos instancias que son propias de las políticas sociales: diseño e implementación (Giménez Mercado y Valente Adarme, 2010).

En 2011, la Organización de Estados Americanos (OEA) aprobó una propuesta de estándares de medición de derechos sociales presentada por el Grupo de Trabajo sobre análisis de los 
informes efectuados por los Estados en el marco del Protocolo de San Salvador. En el documento se expresa:

\begin{abstract}
Se busca estimular un proceso de evaluación y de medición de cumplimiento de derechos sociales que trascienda la idea de mero reporte, sino que se convierta en un instrumento útil para el diseño y evaluación permanente para las políticas públicas al interior de los Estados tendientes a asegurar el cumplimiento de los derechos sociales (p. 5).
\end{abstract}

De allí que se proponga la aplicación de tales principios en el diseño e implementación de cada política social. Asimismo, tales estándares -a los cuales haremos referencia a continuaciónpueden ser medidos mediante indicadores estructurales o bien de proceso y de resultado. Para obtener una adecuada evaluación de una política social es necesario integrar los tres. Los primeros miden la incorporación formal de tales principios en los Estados. Por su parte, los indicadores de proceso tienen en cuenta las acciones en curso de parte de cada país para la implementación de los derechos sociales. Ya por último los indicadores de resultado procuran reflejar los logros obtenidos por cada Estado en la realización de los derechos sociales por medio de sus programaciones públicas.

El primero de los estándares propuestos es el de universalidad, o inclusión en términos propios de las estrategias de desarrollo, por medio del cual se propone la implementación de programas sociales con alcance universal y, por consiguiente, no focalizados (Pautassi, 2010). Los programas con focalizaciones poco flexibles, con algunas excepciones, han abundado en los últimos quince años entre las estrategias de reducción de la pobreza, tanto en nuestra región como en el país (Stampini y Tornarolli, 2012). Si bien no podemos negar los efectos positivos de tales políticas sobre la disminución de la pobreza, optar por políticas universales garantiza el cumplimiento de mínimos comunes irrenunciables en materia de derechos sociales, y mayores garantías de igualdad y no discriminación (Abramovich y Pautassi, 2009). En tal sentido, Lo Vuolo (1995) ha propuesto la implementación de un ingreso ciudadano básico previsto para todos los habitantes argentinos y cubierto con la riqueza socialmente creada.

En los últimos tiempos se ha producido una interesante discusión en torno al binomio universalismo-focalización. Algunos cientistas sociales señalan que la última comprende estrategias oportunas para seleccionar potenciales destinatarios de las políticas sociales entre quienes se encuentran con menores necesidades básicas satisfechas. Al mismo tiempo, tal estrategia evitaría el desperdicio de los recursos destinados al área (Grosh, 1994; Repetto, 2010; Mazzola, 2012). Contra aquel mito del significativo gasto social que conllevaría la 
implementación de programas sociales universales, Pautassi (2015) afirma que ello no es cierto por cuanto el incremento en el gasto social no sería tal y, asimismo, se reducirían desigualdades sociales de género, siendo aquella el área en la que se ubican actualmente las principales brechas de bienestar.

El otro estándar aplicable es el que propone el máximo de la utilización de los recursos disponibles, el cual surge de la interpretación del artículo 2.1 del Pacto Internacional de Derechos Económicos, Sociales y Culturales. Si bien en dicho pacto se reconoce que los Estados no pueden satisfacer de manera inmediata el cúmulo de derechos humanos reconocidos, surgen claramente dos principios: la obligación de adoptar medidas concretas para la satisfacción de los derechos y el uso de la mayor cantidad de recursos de los que dispone. Asimismo, tal principio se encuentra particularmente receptado en el Protocolo Adicional a la Convención Americana sobre Derechos Humanos en materia de derechos sociales que fue ratificado por nuestro país en 2003. En una jurisprudencia argentina de $2008-$ proveniente de la Cámara Nacional de Apelaciones- ante una acción de amparo presentada por una asociación civil, el tribunal condenó al Gobierno de la Ciudad de Buenos Aires a que amplíe los recursos destinados al área de educación a fin de garantizar vacantes en el nivel inicial. Desde 2002, dicho Gobierno incumplía con sus obligaciones constitucionales al realizar una sub ejecución presupuestaria equivalente al 30\% del monto destinado a educación, de allí que en la condena se mencionara el estándar del máximo de la utilización de los recursos disponibles. Cuando la causa llegó al Superior Tribunal de Justicia en 2011, las partes arribaron a un acuerdo en donde el Gobierno se comprometía a llevar a cabo las obras de infraestructura necesarias a fin de garantizar las vacantes faltantes. A pesar de aquel compromiso, fue en 2014 que el Poder Ejecutivo presentó un presupuesto, aplicable a 2015, que no comprendía los recursos necesarios para el progreso de las obras, lo cual motivó una queja de parte de la asociación civil. La decisión del Tribunal fue intimar durante un plazo de diez días al Gobierno a fin de que realice las explicaciones correspondientes. Finalmente, transcurridos sesenta días -que incluyeron protestas sociales- se realizó el incremento en la partida presupuestaria. Sin embargo, la falta de vacantes en el nivel inicial continúa siendo un grave inconveniente en la Ciudad de Buenos Aires (2).

El principio de participación es otro de los estándares provenientes del derecho internacional de los derechos humanos aplicable a las políticas con fines redistributivos. Mucho ha insistido la bibliografía sobre el tema en la importancia de la incorporación de la percepción de los sujetos, ya sea como mediadores en la prestación de los servicios o en la construcción de indicadores cualitativos, en los procesos de formulación de programas sociales (Franco, 2006; 
La Serna, Ase, Burijovich, Echavarri, Moreira Slepoy y otros, 2010; Tonon, 2010). Para el enfoque de derechos tal cuestión deja de ser un punto de discusión en el ámbito de dichos programas y se convierte en una exigencia estatal, la cual, debe garantizar la incorporación de la participación de los destinatarios en las diferentes etapas de las políticas sociales. En contra de aquellos mitos que señalan lo costoso -en términos de recursos económicos- de proponer instancias de participación ciudadana, el enfoque propone que el Estado brinde condiciones necesarias para el cumplimiento de derechos ya establecidos. No se trata solo de generar nuevas instancias de participación sino principalmente de ofrecer las oportunidades para concretar las ya existentes, tales como la iniciativa popular, la libertad sindical, el derecho de asociación y de reunión, la libertad de expresión, el acceso a la información (Abramovich, 2006). Aunque tampoco es menos cierto la necesidad de trabajar en el ámbito de la construcción de las subjetividades y mediante la difusión y apropiación de tales derechos, lo que algunos autores denominan empoderamiento, propiciar en los ciudadanos el aprovechamiento de tales instancias que no son más que el ejercicio de sus propios derechos (Pautassi, 2010; Becher, 2017). El Achkar (2008) afirma respecto de quienes realizan educación popular en derechos humanos: “... estamos haciendo trabajo cultural en tanto estamos haciendo el esfuerzo de impactar en las percepciones, relaciones sociales y sentidos comunes" (p. 14).

Que existan estos derechos no supone que los Estados realizan un cumplimiento estricto de ellos en sus acciones estatales. De hecho, la historia argentina no da muestras de tal cumplimiento. Por ello podemos preguntarnos: ¿cuál es el estándar previsto para colocar límites al decisionismo estatal? Si bien el enfoque de derechos humanos no pretende limitar las decisiones estatales, no por ello deja de procurar que el Estado cumpla con las obligaciones que ha adquirido ante organismos internacionales. En tal sentido, propone como principio el de acceso a la justicia y mecanismos de reclamo. Ello supone que tanto ante la negatoria o bien el incumplimiento de algunas de las prestaciones de los programas sociales se puede acceder a instancias de reclamo para el cumplimiento de las mismas. Vale señalar que no solo comprende las instancias jurisdiccionales sino también las administrativas. De allí que quedan comprendidos aquellos espacios de reclamo que son propios de los organismos del Poder Ejecutivo -tales como (en el mejor de los casos) la Administración Nacional de la Seguridad Social (ANSES)- donde se ejecutan las políticas. El Estado, tal como establece la Comisión Interamericana de Derechos Humanos (2008), tiene como principal obligación la de remover todos aquellos obstáculos que obturen un efectivo acceso a la justicia. Asimismo, se agregan otras condiciones como las de garantizar el debido proceso y la posibilidad de iniciar reclamos 
urgentes o bien remedios cautelares o preventivos. Ello supone de parte del Estado prever y disponer todas las modificaciones institucionales -con los recursos que conlleva- para lograr la garantía de tales principios en materia de acceso a la justicia.

\section{¿Qué particularidades agrega la cuestión de las juventudes?}

En primer lugar, vamos a comentar nuestra propuesta sobre el modo de comprender a quienes integran el colectivo juvenil. La idea es definir a dicho colectivo no solo a partir de criterios etarios, los cuales son comunes en materia jurídica, sino incorporar también a los culturales. No es lo mismo ser joven y residir en la Ciudad de Buenos Aires o en una provincia del interior, o ser joven y pertenecer a un sector socioeconómico elevado o a uno bajo, o ser joven mujer o varón. Esta propuesta comprende en la conformación de la juventud un criterio etario y otro social, en donde la consideración de las desigualdades sociales adquiere relevancia. Ello nos muestra que no existe un modelo único de juventud y de allí la propuesta de pluralizar el término (Margulis, 2015). Epistemológicamente tales consideraciones son propias de un enfoque socio-antropológico.

En cuanto al enfoque de derechos y ante el decisionismo estatal en materia de políticas sociales, las juventudes son comúnmente destinatarias de programaciones públicas a partir de lo que el Estado, respondiendo a estereotipos sobre el colectivo, ha considerado apropiado para ellos (Chaves y Faur, 2006). Allí es donde el principio de participación adquiere una particular significancia por cuanto se convierte en la instancia oportuna para conocer las necesidades juveniles. El tipo ideal para tales políticas -como señala Balardini (2000)- es el de programas en donde las acciones sean imaginadas y diseñadas por los mismos jóvenes. Sin embargo, lo que ha primado históricamente en nuestro país, tal como advertíamos anteriormente, son políticas en donde las juventudes son interpeladas a procesos de socialización clásicos (tales como el empleo o la educación) considerados apropiados para ellos por el Estado. Si bien los problemas sociales de los miembros del colectivo sociogeneracional tienden a ubicarse en el ámbito del empleo y la educación, no deben ser considerados como factores aislados de los propiamente culturales. A partir de tales consideraciones, la inversión social del Estado debería intentar redistribuirse mejor de modo que el empleo continúe siendo un área de inversión importante, pero no se desatiendan otras instancias de inclusión social que también contribuyen favorablemente a la futura inserción de las juventudes. 
En el ámbito propio del enfoque de derechos humanos en intervenciones dirigidas a las infancias se han incorporado los estándares que surgen de la Convención Internacional de los Derechos del Niño. Entre tales estándares destacan el interés superior del niño o bien el de expresar su opinión y ser escuchado (Pautassi y Royo, 2012). No dejamos de lado tales estándares, pero tal como hemos señalado nos ubicamos en el plano de las políticas sociales de juventudes las cuales, tanto desde consideraciones etarias como sociales, se encuentran en una instancia vital y social diferente a la de las infancias. Por ello, la insistencia en el derecho a la participación como un aspecto clave en la construcción de las necesidades sociales y, por consiguiente, su influencia en políticas sociales de juventudes.

\section{Los programas sociales seleccionados}

Tal como señalábamos al comienzo, a fin de reflejar la experiencia argentina -desde ya parcialmente- tendremos en cuenta dos programas sociales con presencia juvenil. Uno de ellos implementado a nivel nacional -el Programa de Respaldo a Estudiantes Argentinos (PROGRESAR)- y otro en la provincia de San Luis: el Plan de Inclusión Educativa (PIE). También como ya hemos mencionado, nos parece significativa la incorporación de un programa de una provincia del interior de Argentina, de similares características al PROGRESAR, por cuanto no abundan los estudios sobre el tema en dichas zonas geográficas. Asimismo, tal circunstancia agrega una serie de particularidades tanto en su diseño como en su implementación.

El Programa de Respaldo a Estudiantes Argentinos consiste en una transferencia de ingresos condicionada, por medio de la cual se otorga a sus destinatarios -jóvenes de entre 18 y 24 años desempleados o empleados informalmente- una prestación dineraria a cambio del cumplimiento de una condicionalidad educativa, ya sea en los niveles primario, secundario o universitario. Al mismo tiempo, se agrega una condicionalidad de salud que exige de parte de los receptores la asistencia anual a centros de salud públicos. El programa inició su implementación a fines de 2014 por medio del dictado del Decreto Nro 84/2014 y continúa vigente hasta la actualidad.

Los datos actuales sobre la cantidad de destinatarios del PROGRESAR -que cubren hasta junio del año 2016- señalan que cuenta con un total de 723.085 receptores. La edad en la que se concentra la mayor cantidad de destinatarios es la de 19 años y la mayoría de ellos asiste al nivel secundario seguido por terciario y universitario (3). 
El Plan de Inclusión Educativa es una transferencia de ingresos condicionada que exige a sus destinatarios el cumplimiento de una condicionalidad educativa en los niveles primarios o secundarios de la educación formal. Dado que el fin es la terminalidad educativa pueden acceder al primer nivel los jóvenes a partir de los quince años y al segundo a partir de los veinte años. Si bien el programa no establece límites etarios -de modo que pueden acceder ciudadanos de diferentes edades- lo que predomina es la presencia de integrantes del colectivo de entre 15 y 25 años (http://www.ulp.edu.ar). Dicha acción estatal inició su implementación en 2015, por medio de la Ley Nro. II 0911 2014, y tiene vigencia hasta la actualidad.

A continuación, proponemos describir el modo en que se presentan los estándares del derecho internacional de los derechos humanos, a los cuales hicimos referencia anteriormente, en cada uno de esos programas sociales.

\section{Precisiones metodológicas}

En la introducción mencionamos que los aspectos cualitativos de este texto se insertan en un trabajo de campo correspondiente a una tesis doctoral en curso, cuya investigación se desarrolla en el marco del proyecto "culturas juveniles" de la UNSL. En el marco de dicho trabajo se han analizado los documentos oficiales ligados a los programas y realizado entrevistas semi estructuradas y grupales con los jóvenes destinatarios, quienes tienen una edad promedio de 22 años. Al mismo tiempo, la mayoría de ellos son estudiantes universitarios en la UNSL y en la Universidad Nacional de Villa Mercedes (UNViMe). En cuanto a la identidad sexual, el diseño muestral se encuentra equiparado entre varones y mujeres, perteneciendo la mayoría de ellos a estratos socioeconómicos bajos ubicados en el primer quintil de ingresos per cápita familiares. Esas mismas características socioeconómicas son las que permiten el ingreso a los programas pues, tal como veremos, entre sus criterios de selección se encuentran presente tales aspectos.

Los documentos y las entrevistas -que ascienden a un total de treinta individuales y cuatro grupales- fueron analizados de acuerdo con los criterios que son propios de las investigaciones cualitativas. En una primera instancia se fueron realizando comentarios generales que iban surgiendo de la lectura del material empírico, en relación con el marco de interpretación propio del investigador. En una segunda etapa se identificaron los temas y subtemas centrales en torno a los cuales se agrupa una importante cantidad de información. Finalmente se seleccionaron los ejes que organizan la redacción del informe final. En esta oportunidad, 
hemos seleccionado dos subtemas que se vinculan con aspectos relativos a los derechos humanos y las políticas sociales.

\section{El diseño de los programas}

En cuanto al estándar de universalidad, los programas no lo cumplen pues son focalizados. El PROGRESAR sólo comprende entre sus destinatarios a jóvenes de entre 18 y 24 años desocupados o bien que se desempeñen en la economía informal pero no superen el salario mínimo. Desde ya, esto último es bastante difícil de comprobar pues quienes se desempeñan en el marco de dicha economía no cuentan con registros formales ante ANSES. Quienes no cumplen con esos requisitos no pueden ingresar al programa.

Por su parte, el PIE divide sus criterios de selección en acceso al nivel primario y secundario de la educación formal. En el primer caso se excluye a aquellos jóvenes que tengan menos de 15 años y en el segundo a los de menos de 20. Los que se encuentran entre 16 y 19 años pueden ingresar al nivel secundario solo cuando tengan una discontinuidad mayor de dos años en su trayectoria escolar del nivel de educación secundaria. Las fechas se computan al 31 de diciembre de 2014 y los que cumplan las edades requeridas por fuera de ella no pueden ingresar al sistema.

Vale señalar que una modificación introducida en 2015 en el Programa de Respaldo a Estudiantes Argentinos -por medio del Decreto Nro. 505/15- elevó el mínimo de un salario a tres, pero cuenta no solo los ingresos del destinatario sino los de todo el grupo familiar. Ello coloca una importante restricción para las juventudes y la posibilidad de adquirir independencia respecto de su contexto familiar. Asimismo, en ambos programas, se establecen una serie de requisitos formales que pueden conducir a excluir a potenciales destinatarios, ya sea porque no cuentan con el Documento Nacional de Identidad o una residencia mínima de cinco años en el país o de dos años en la provincia de San Luis. En tal caso debemos recordar siempre que se trata de programas sociales dirigidos a poblaciones en condiciones de vulnerabilidad social, tanto material como simbólica.

El estándar de participación es otra de las grandes ausencias que presentan ambos programas pues no establecen ninguna instancia, ya sea en lo que fue su etapa de diseño o posterior evaluación, que incorpore la participación de los destinatarios. Ya hemos hecho referencia a la vinculación y la importancia que tiene este aspecto en materia de juventudes. Su incidencia -tal como señala Balardini (2000)- en la construcción de ciudadanías más o menos democráticas también es clave. Si bien es cierto que el Estado argentino de los primeros quince años del 
nuevo siglo ha implementado importantes acciones juveniles participativas, no deja de ser relevante la incorporación de tales instancias en cada programa social, pues ¿cómo conocer sino las necesidades propiamente juveniles? Ello nos muestra que los programas no incorporan la perspectiva de las juventudes en la construcción de las necesidades que pretenden satisfacer.

En lo referido al acceso a la justicia y los mecanismos de reclamo, el PROGRESAR presenta una importante modificación respecto de programas sociales anteriores: la de su implementación en el ámbito de la Administración Nacional de la Seguridad Social. Ello equipara a los destinatarios del programa en lo relativo al acceso a procesos recursivos de la administración pública propios de quienes se desempeñan o se han desempeñado, en el caso de jubilados y pensionados, en el marco del empleo formal. A diferencia de dicho programa, el PIE se implementa dentro del ámbito del Ministerio de Educación lo cual lo ubica institucionalmente en el plano de la protección social.

Si bien estos programas, a luz de los estándares internacionales, presentan las deficiencias que hemos señalado, no podemos dejar de mencionar su significativa diferencia respecto de las políticas neoliberales de la década de 1990. Quizá algunos consideren innecesaria la comparación, pero en materia de políticas sociales no tiene desperdicio -tal como propone Esping-Andersen (1990)- la posibilidad de comparar la calidad de los derechos sociales, y por consiguiente las políticas propuestas en cada modelo de régimen de bienestar. En los años noventa, los programas que primaron proponían estrictos criterios de focalización, de modo que el acceso a los mismos era sumamente dificultoso. Al mismo tiempo que las prácticas clientelares funcionaban como los principales mecanismos de engranaje en el vínculo entre las políticas y sus destinatarios. Allí eran los caudillos provinciales quienes adquirían protagonismo en el número de políticas que se implementaban y los recursos que se destinaban para las mismas en cada provincia. En ningún caso se incorporaron mecanismos de participación ciudadana, no en los diseños y menos aun en las instancias de monitoreo, como así tampoco acciones estatales destinadas a incentivar dicha participación. Todos los programas de aquella década se implementaron en el marco de los ministerios públicos, limitando posibilidades de acceso a la justicia y mecanismos de reclamo, favoreciendo condiciones de posibilidad para la emergencia de redes clientelares (Franco, 2006; Hintze, 2006).

\section{Algunos aspectos de su implementación desde la perspectiva de los destinatarios}


En materia de políticas sociales una preocupación constante es la del acceso a información sobre las prestaciones de los programas, lo cual se vincula con la posibilidad de lograr mayor universalidad o alcance en la instancia de implementación. Bien pueden las acciones estatales diseñarse con fines universales, pero en su ejecución encontrar obstáculos que obturen dicho acceso. De modo que este fue uno de los aspectos incorporados en nuestra investigación. De allí que pudimos advertir que las juventudes sanluiseñas que integraron la muestra acceden a información sobre los programas, ya sea para el acceso al sistema o consulta sobre trámites posteriores, por medio de la red social Facebook. Dicha red social -tal como muestra la Encuesta Nacional de Consumos Culturales y Entorno Digital- ha adquirido una particular centralidad entre las opciones de relaciones virtuales para los jóvenes.

"Yo por ejemplo me enteré por Facebook" (A. 22 años, destinataria del PROGRESAR).

"Por Facebook, porque había una campaña muy fuerte y subían la información y entré, creo que era uno de los noticieros que tienen página y me derivaba a la página de ANSES y ahí estaba la información" (V. 21 años, destinataria del PROGRESAR).

Sin embargo, tales redes tienen una presencia menor en el caso de los destinatarios del programa provincial. A partir de ello, surgen -para ambos destinatarios- las redes de sociabilidad como un medio por el cual obtienen acceso a información sobre las acciones estatales:

"P (pregunta): Primero te pregunto ¿Cómo te enteraste que el plan existe?

$\mathrm{R}$ (respuesta): Me avisó mi madre y ella escuchó en la radio" (F. 23 años, destinatario del PIE).

"Yo me enteré por mi primo, él me dijo" (E. 25 años, destinatario del PIE).

"Yo me enteré por mi abuelo" (R. 21 años, destinataria del PIE).

“... y mi amigo ya lo tenía y su tía trabajaba en ANSES, entonces ella me dijo cómo era... todos los trámites, todas las cosas, ahí me averigüé bien" (N. 20 años, destinatario del PROGRESAR).

Al mismo tiempo, en el relato de $\mathrm{N}$ es posible identificar que las redes de sociabilidad no sólo constituyen un medio de acceso a información, sino también un recurso provechoso frente a posibles dificultades que pueden surgir en el marco del programa, aunque ese contacto no ocupe un rango de jerarquía dentro de la infraestructura de la organización: 
"Yo el problema que estoy teniendo con la tarjeta me dijo el del banco que vaya a ANSES y un amigo me dice que tengo que sacar turno; el turno lo tengo que sacar para dentro de bastantes días y yo necesito la plata ahora... entonces yo le pregunté a ella y ella va a hacer la consulta, no es que me va a gestionar porque no es administradora, es la empleada que limpia..." ( $\mathrm{N}$, comunicación personal, 25 de octubre de 2016).

Tanto entre varones y mujeres las redes de sociabilidad adquieren una relevante significancia en el marco de sus actividades en los programas. Sin embargo, y ello surge de los propios relatos juveniles, son las mujeres quienes cuentan con menores soportes de sociabilidad, pues dichos soportes provienen de experiencias de socialización previas de las cuales las mujeres no han participado por tener que ocuparse de tareas de cuidado, ya sea respecto de sus propios hijos o de otros en el marco de empleos informales.

Con menor presencia pero no significancia aparecen en escena los medios de comunicación. Entre las juventudes destinatarias de los programas son el discurso de la ex presidenta Cristina Fernández, en mayor medida y entre quienes reciben el programa social nacional, y el del gobernador Alberto Rodríguez Saá, en menor medida y entre quienes reciben el programa provincial, recursos por medio de los cuales han accedido a información sobre las acciones estatales, como así también sobre los requisitos necesarios para acceder a las prestaciones. Los jóvenes lo expresan del siguiente modo:

"Por los medios de comunicación, cuando habló la presidenta" (L. 21 años, destinataria del PROGRESAR).

"Yo igual, el día que Cristina sacó el discurso" (A. 22 años, destinataria del PROGRESAR).

"P: pero en televisión ¿qué estabas viendo? R: A la presidenta..." (S. 22 años, destinatario del PROGRESAR).

“También, lo mismo que los chicos, me enteré cuando la presidenta lo anunció..." (An. 23 años, destinataria del PROGRESAR).

"Yo me enteré porque viste que salió un anuncio de que iban a largar un plan... lo escuché en la radio... y después lo anunció el gobernador" (La. 26 años, destinataria del PIE). 
"P: ¿Cómo te enteraste que existe el Plan de Inclusión Educativa? R: Por la tele" (M. 25 años, destinatario del PIE).

"P: ¿Qué fue lo que escuchaste? R: El noticiero... el gobernador dijo que iba a sacar un plan social para la gente..." (D. 24 años, destinataria del PIE).

Si bien los medios de comunicación adquieren el protagonismo que comentábamos, las redes de sociabilidad no pierden eficiencia como uno de los medios predilectos para acceder a información sobre los programas y sus requisitos, por cuanto tal como comentan las mismas juventudes que señalaron haber conocido el programa por medio de los discursos "me hice averiguar con un amigo" (N. Destinatario del PROGRESAR); "uno ya lo tenía, en la comunicación con los amigos" (A. Destinataria del PROGRESAR) o que "por comentarios de la gente" ya sabía de la posible existencia del plan (D. Destinataria del PIE).

Ante la necesidad de efectuar reclamos, los jóvenes deben acudir a los organismos públicos nacionales o provinciales previstos a tal fin. Dado que el material empírico al que acudimos proviene de actividades de investigación desarrolladas a partir de 2016 en adelante, surgen en los comentarios aspectos sobre el Programa de Respaldo a Estudiantes Argentinos vinculados con la nueva coyuntura sociopolítica argentina. Por ello, ante denegatorias en el acceso a las prestaciones los miembros del colectivo socio-generacional debieron acudir a instancias de reclamo en la Administración Nacional de la Seguridad Social. A partir de tales experiencias califican a los agentes estatales con el conocido estereotipo de "típicos empleados públicos" (A). Por su parte, $\mathrm{V}$ comenta que el trato no es agradable, pues menciona que tuvo que concurrir en reiteradas oportunidades a ANSES para completar los requisitos de ingreso al programa, como así también para gestionar reclamos:

"Te tratan como si no supieras lo que tenés que hacer o cuáles son tus derechos. Después cuando vas un par de veces y se dan cuenta de que no van a llegar a buen puerto por ahí, te dan las consultas, la mayoría de las veces es de mala manera, hasta que en mi caso, no sé, se habrán acostumbrado, se habrán resignado, que ya después me atendían bien y lo intentaron solucionar. Calculo que para que ya no vaya más" (risas). (V, comunicación personal, 25 de octubre de 2016).

Asimismo, comentan que frente a posibles dificultades que puedan surgir en el marco del programa no cuentan con un funcionario público que los oriente. En tal caso, la mayoría menciona que conoce a algún amigo que puede guiarlos. De allí que las redes de sociabilidad con que cuentan las juventudes se convierten en un capital que no solo posibilita el acceso a 
información sobre los programas sino también a las prestaciones. Ya anteriormente $\mathrm{N}$ nos había contado que cuando tenía inconvenientes vinculados al programa acudía a su tía -An también lo hizo- $y$ ahora nos comenta que también conoce a una amiga que lo ayuda:

"P: Si tienen alguna dificultad en el programa ¿Hay alguna persona a la cual ustedes puedan concurrir para consultarle?

R: Yo el problema ahora que tengo, primero tenía mi tía, pero ahora tengo una amiga que justo trabaja en ANSES... y yo busco mis amigos de ANSES" (risas) (N).

"El año pasado teníamos una conocida que por cualquier cosa vamos a ella" (An).

En el caso del Plan de Inclusión Educativa, la proximidad con la burocracia y por consiguiente con las instancias de reclamo es diferente:

"Yo llegué y le dije que me habían mandado acá, así que entré de suertudo nomás, me quedaba cerquita, porque vivo acá nomas, porque estamos a 7 cuadras, así que bueno, fue suerte nomás" (T. 22 años, destinatario del PIE).

"P: ¿Y cómo fue retomar? Vos te tuviste que ir a otra provincia y ¿cómo fue el regreso al plan?

R: Yo agarré y entré de cara dura, "mire, acá me llamaron" no sé cómo le dije, que tenía que empezar a cursar y "siéntese nomas" y...

P: El trato fue con el docente" (asiente) (E. 25 años, destinatario del PIE).

A diferencia del programa nacional, las juventudes se encuentran con una estructura administrativa que les es más amena dado que pertenece a su misma provincia. Ello supone enfrentarse a una burocracia con la cual ya han tenido algún contacto en el marco de sus trayectorias sociales y, por consiguiente, conocen los diferentes circuitos administrativos que le son propios, como así también al personal y los espacios en donde desempañan sus tareas. De parte de los agentes estatales ello permite que puedan facilitar instancias para el acceso a determinadas prestaciones o respuesta a reclamos sin depender de otro nivel de gobierno. Al mismo tiempo, dichos funcionarios pueden estar al tanto de las condiciones juveniles de quienes residen en la provincia y adaptar aspectos del programa a partir de esas circunstancias. Por ello, muchos destinatarios ingresaron o retomaron el programa simplemente concurriendo a las aulas y en el contacto con los docentes. Sin dudas, no son menores las distinciones entre una y otra acción estatal, pues en un caso el contacto cercano de los jóvenes son docentes universitarios que no tienen ningún tipo de involucramiento con la estructura del programa y, en el otro, docentes de nivel medio que reciben un estipendio del 
Estado para desarrollar contenidos al grupo de destinatarios. Además de ello, en el Plan de Inclusión Educativa, a diferencia del programa nacional, un funcionario estatal concurre con una frecuencia mensual a fin de entablar un contacto con los jóvenes, lo cual les permite comentar sus experiencias en el marco del Plan.

\section{Conclusión}

A inicios del siglo XXI, con la Declaración de los Objetivos del Milenio, se propuso la incorporación del marco de los derechos humanos en las estrategias de desarrollo. Entre tales estrategias y con los fines que hemos descripto quedan comprendidas las políticas sociales. Quince años después, cuando se revisa el cumplimiento de aquellos Objetivos, continúa presente la propuesta realizada en aquel entonces. Desde los años 2000, el enfoque de derechos comienza a emerger como una perspectiva de análisis de las intervenciones sociales que al mismo tiempo que refiere a las obligaciones estatales internacionales conlleva un conjunto de supuestos conceptuales y metodológicos. A partir de ello, tal como hemos señalado, se introducen modificaciones en el modo de concebir a las políticas sociales y propuestas de estándares para su estudio. Tales estándares surgen de los instrumentos internacionales de derechos sociales y civiles, y de la labor de los grupos de trabajo, en la medida en que establecen principios aplicables a las acciones estatales con fines redistributivos. Entre tales principios, el de universalidad contribuye a la reducción de desigualdades sociales por cuanto significa mayores garantías de igualdad y no discriminación. Asimismo, aporta a la eliminación de brechas de género en donde se ubica una de las principales disparidades en el régimen de bienestar argentino de las últimas décadas.

Por otra parte, esta propuesta -que involucra obligaciones estatales internacionales- coloca límites al decisionismo político. No se trata de limitar la autonomía propia de la soberanía estatal en la decisión de la estrategia de desarrollo que decida llevar a cabo, sino de establecer los principios que deberían guiar tales procesos a partir de los compromisos en materia de derechos humanos. Es el poder judicial, con mayor preponderancia, pero también los organismos administrativos de reclamo dentro del ámbito del Poder Ejecutivo, los encargados de realizar el control del contenido de los programas sociales. Desde ya, no es su función implementar políticas sociales, pues lo que les corresponde es señalar aquellos aspectos en los que tales políticas se distancian de los estándares del derecho internacional de los derechos humanos y establecer las correspondientes responsabilidades. 
En materia de políticas de juventudes es el derecho de participación el que adquiere una particular significación, pues se trata de un colectivo con necesidades particulares las cuales solo podrán conocerse si se los incorpora en las instancias de diseño. Si bien esto no es solamente propio del colectivo juvenil, en el análisis de los diferentes estándares ante la formulación de políticas dirigidas a sectores sociales específicos, la incorporación de su participación deviene central.

En cuanto a los casos de programas sociales seleccionados, podemos advertir el modo en que funcionan los estándares de derechos humanos. A partir de describir su diseño, es posible apreciar que ambas acciones estales son focalizadas, lo cual implica no cumplir con el estándar de universalidad. De las dos, el programa provincial contiene criterios de focalización más estrictos -lo cual afecta su masividad- pues solo permite el ingreso a los ciudadanos entre las edades establecidas, y que la hayan cumplido antes de una determinada fecha. Al mismo tiempo, ninguno de los dos programas cuenta con instancias de participación de la población a la que se dirigen, y esto afecta la consideración de necesidades y particularidades propias de esos destinatarios. En cuanto a los mecanismos de reclamo, el Programa de Respaldo a Estudiantes Argentinos se implementa en el ámbito de la Administración Nacional de la Seguridad Social destinado a los trabajadores en condiciones de formalidad. Dicha incorporación resulta significativa pues promueve mayores niveles de formalización y entre los destinatarios un imaginario que los equipara a los trabajadores del sector formal con acceso a las mismas instancias recursivas. En lo referido a la implementación de los programas solo nos centramos en algunos aspectos desde la perspectiva de los receptores. Entre tales aspectos, los ligados al acceso a información sobre los programas, y por consiguiente sus prestaciones, y los mecanismos de reclamo. En la primera dimensión adquieren un protagonismo central las redes de sociabilidad -aunque las virtuales también tienen su espacio- tanto en lo referido al acceso a la información sobre las acciones estatales, como así también sobre dificultades que pueden ir surgiendo a partir del ingreso al sistema. En el acceso a mecanismos de reclamo, los jóvenes que deben contactarse con ANSES comentan mayores dificultades e inconvenientes en el trato recibido de parte de los agentes estatales. A diferencia de ello, quienes se vinculan con la burocracia provincial identifican claramente a los encargados del programa y no comentan dificultades ante diversos trámites que deben realizar. Sin dudas, aspectos relacionales y territoriales entran en juego en las diferencias entre uno y otro programa, pues quienes deben acudir a la estructura nacional encuentran mayores dificultades que quienes lo hacen con la provincial. Dado que ya conocen los circuitos administrativos locales e incluso a los agentes estatales y los espacios en donde desarrollan sus tareas, tal aproximación con la 
estructura administrativa se vuelve más amena. Ello nos muestra que el enfoque de derechos puede prever que la institucionalidad funcione de un determinado modo, pero si no se incorporan aspectos propios de cada contexto y de las condiciones de quienes son sus destinatarios, la institucionalidad pierde eficiencia para lograr mayor alcance y acceso.

\section{Notas}

(1) Tales estándares surgen de diferentes cuerpos normativos internacionales en donde constan las obligaciones asumidas por el Estado argentino, al ratificarlos, en materia de derechos sociales. Si bien son variados los artículos que podemos considerar, proponemos especialmente los siguientes: el artículo 2 del Pacto Internacional de Derechos Económicos, Sociales y Culturales; los artículos 17 a 22 y 26 del Pacto Internacional de Derechos Civiles y Políticos; el artículo 1 de la Convención sobre la Eliminación de Todas las Formas de Discriminación contra la Mujer; el artículo 13 y 29 de la Convención Americana sobre Derechos Humanos. Asimismo, es fundamental considerar en la elaboración de los estándares la labor de la Comisión del Pacto Internacional de Derechos Económicos, Sociales y Culturales y del Grupo de Trabajo sobre análisis de los informes efectuados por los Estados en el marco del Protocolo de San Salvador.

(2) Cámara Nacional de Apelaciones en lo ConAdmFed. "Asociación Civil por la Igualdad y la Justicia contra GCBA s/ amparo (art. 14 CCABA)", 2008.

(3) ANSES Transparencia. Cantidad de destinatarios del Programa de Respaldo a Estudiantes Argentinos. Recuperado de http://www.transparencia.anses.gob.ar/gobierno-abierto/progresar

\section{Bibliografía}

Abramovich, V. (2006). Una aproximación al enfoque de derechos en las estrategias y políticas de desarrollo. Revista de la CEPAL, 88, 35-50.

Abramovich, V. y Pautassi, L. (2009). El enfoque de derechos y la institucionalidad de las políticas sociales. En Abramovich, V. y Pautassi, L. (Comps.). La revisión judicial de las políticas sociales (pp. 279-340). Buenos Aires: Editores del Puerto.

Autés, M. (2004). Tres formas de desligadura. En Karsz, S. (Coord.). La exclusión: bordeando sus fronteras. Definiciones y matices (pp. 15-53). Barcelona: Editorial Gedisa.

Balardini, S. (2000). De los jóvenes, la juventud y las políticas de juventud. Última Década, 13, 11-24.

Becher, Y. (2017). Cartografías socio-estatales y subjetividades. Un recorrido sobre programas sociales en la contemporaneidad. Buenos Aires: Teseo. 
Burchardt, T.; Le Grand, T. y Piachaud, D. (2002). Degrees of exclusion: developing a dynamic, multidimensional measure. In Hills, J.; Le Grand, T. and Piachaud, D. (Eds.). Understanding social exclusion (pp. 30-43). Oxford: Oxford University Press.

Castel, R. (2004). Encuadre de la exclusión. En Karsz, S. (Coord.). La exclusión: bordeando sus fronteras. Definiciones y matices (pp. 55-86). Barcelona: Editorial Gedisa.

Chaves, M. y Faur, E. (2006). Informe: investigaciones sobre juventudes en Argentina. Estado del arte en ciencias sociales. La Plata, Buenos Aires: UNSAM-Ministerio de Desarrollo Social-DINAJU - UNICEF.

Comisión Interamericana de Derechos Humanos-Organización de los Estados Americanos (2008). Lineamientos para la elaboración de indicadores de progreso en materia de derechos económicos, sociales y culturales. Washington D.C.

Danani, C. (2004). El alfiler en la silla: sentidos, proyectos y alternativas en el debate de las políticas sociales y de la Economía Social. En Danani, C. (Comp.). Política social y economía social: debates fundamentales (pp. 9-27). Buenos Aires: UNGS-Fundación OSDE-Editorial Altamira.

De Gaulejac, V. y Taboada-Léonetti, I. (1994). La Lutte des places. París: Desclée de Brouwer.

Dubet, F. (2011). Repensar la justicia social. Contra el mito de la igualdad de oportunidades. Buenos Aires: Siglo XXI Editores.

El Achkar, S. (2008). Ejercicio de la ciudadanía: el desafío educativo de nuestro tiempo. Venezuela: Universidad Central de Venezuela.

Esping-Andersen, G. (1990). The tree worlds of welfare capitalism. Cambridge: Polity Press.

Estivill, J. (2003). Concepts and strategies for combating social exclusion. Geneva: International Labour Office.

Franco, R. (2006). Modelo de política social en América Latina en el último cuarto de siglo. En Franco, R. y Lanzaro, J. (Coords.). Política y políticas públicas en los procesos de reforma de América Latina (pp. 147-167). Buenos Aires: Miño y Dávila.

Giménez Mercado, C. y Valente Adarme, X. (2010). El enfoque de los derechos humanos en las políticas públicas: ideas para un debate en ciernes. Cuadernos del Cendes, 27(74), 51-79.

Grosh, M. E. (1994). Administering Targeted Social Programs in Latin-America: From Platitudes to Practice. Washigton D.C.: World Bank.

Hintze, S. (2006). Políticas sociales argentinas en el cambio de siglo. Conjeturas sobre 10 posible. Buenos Aires: Espacio Editorial. 
La Serna, C.; Ase, I.; Burijovich, J.; Echavarri, L.; Moreira Slepoy, J. y otros (2010). Entre el intervencionismo y el mercado ¿Qué tipo de Estado abonan las políticas sociales del nuevo siglo? En La Serna, C.; Peón, C. y Ase, I. (Comps.). Frente a la crisis ¿Qué hacer con el Estado? (pp. 195-245). Córdoba: Ediciones IIFAP.

Lenoir, R. (1974). Les exclus. Un français sur dix. París: Le Seuil.

Levitas, R. (2006). The concept and measurement of social exclusion. In Pantazis, C.; Gordon, D and Levitas, R. Poverty and social exclusion in Britain (pp. 123-160). Bristol: The policy press.

Lo Vuolo, R. (1995). A modo de presentación: los contenidos de la propuesta del ingreso ciudadano. En Contra la exclusión: propuesta del ingreso ciudadano (pp. 17-47). Buenos Aires: Ciepp-Miño y Dávila.

Margulis, M. (2015). Juventud o juventudes. Dos conceptos diferentes. Voces en el Fénix. La Revista del Plan Fénix, 6(51), 6-13.

Mazzola, R. (2012). Nuevo paradigma: la Asignación Universal por Hijo en Argentina. Buenos Aires: Prometeo Libros.

Organización de los Estados Americanos (2011). Indicadores de Progreso para Medición de Derechos Contemplados en el Protocolo de San Salvador. Washington D.C.

Pautassi, L. (2007). ¡Cuánto trabajo mujer! El género y las relaciones laborales. Buenos Aires: Capital Intelectual.

Pautassi, L. (2010). Indicadores en materia de derechos económicos, sociales y culturales. Más allá de la medición. En Abromovich, V.y Pautassi, L. (Comps.). La medición de derechos en las políticas sociales (pp. 1-87). Buenos Aires: Del Puerto.

Pautassi, L. (2015). Introducción: situando el bienestar, identificando brechas. En Pautassi, L. y Gamallo, G. (Comps.). El bienestar en brechas: las políticas sociales en la Argentina de la posconvertibilidad. Buenos Aires: Biblos.

Pautassi, L. y Royo, L. (2012). Enfoque de derechos en las políticas de infancia. Indicadores para su medición. N.U. Santiago de Chile: CEPAL.

Repetto, F. (2010). Protección social en América Latina: la búsqueda de una integralidad con enfoque de derechos. Revista del CLAD Reforma y Democracia, 47, 1-24.

Stampini, M. y Tornarolli, L. (2012). The growth of conditional cash transfer in Latin America and the Caribbean: did they go too far? Policy Brief. Inter-American Development Bank, 185, 2-30.

Tonon, G. (2010). La utilización de indicadores de calidad de vida para la decisión de políticas públicas. Polis-Revista de la Universidad Bolivariana, 9(26), 361-370. 
Towsend, P. (1979). Poverty in the United Kingdom. Harmondsworth: Penguin. 